\title{
Ueber Licht- und Farbenperception niederster Organismen.
}

\author{
Von
}

\section{Th. W. Engelmanm.}

Seit lange kennt man einfachste Organismen, deren Bewegungen durch Licht beeinflusst werden. Meist sind es grüne oder doch farbige, mittelst Wimpern oder in anderer Weise aktiv, frei in Wasser bewegliche Formen (Infusorien, Schwärmsporen von Algen, Diatomaceen, Oscillarineen, einzelne Bakterien); aber auch einige farblose (Plasmodien von Myxomyceten, Pelomyxa, Gameten von Chytridium vorax) zeigen die Erscheinung.

Der Einfluss des Lichtes kann sich in sehr verschiedener Weise äussern: Richtung, Geschwindigkeit, Form, Dauer der Bewegung können sich ändern. Dabei pflegen Intensität und Qualität der Reaktion zu variiren einerseits mit Art, Stärke und Richtung des einfallenden Lichtes, andererseits mit der Art des Organismus, auch mit dem Individuum und der Entwicklungsphase in der dieses sich befindet.

Zahlreiche Uebergangsformen verbinden diese Erscheinungen auf der einen Seite mit den verschiedenen photokinetischen Wirkungen, die man bei höheren Pflanzen beobachtet (Form- und Ortsveränderung der Chlorophyllkörner, heliotropische, nyetitropische Bewegungen u. s. w.), auf der anderen mit dem specifischen Process der Lichtperception im Sehorgan der höheren Thiere.

Die weitreichende Bedentung einer umfassenden, eindringenden Erforschung dieser Erscheinungen braucht unter diesen Umständen nicht betont zu werden. In physiologischer wie in phylogenetischer Beziehung verspricht sie gleich werthvolle Aufschlüsse. Inzwischen ist bis heute mit einer solchen Untersuchung nur erst insoweit ein Anfang gemacht, als man Thatsachen zu sammeln 
und zu beschreiben begonnen hat. Eine näbere Einsicht in die Art und den ursächlichen Zusammenhang der Processe vermittelst welcher das Licht die betreffenden Reaktionen veranlasst, fehlt noch durchaus. Jeder Beitrag in dieser Richtung darf desshalb wohl willkommen heissen.

Auf den folgenden Seiten wünsche ich einige Thatsachen mitzutheilen, welche beweisen, dass das Licht auf wenigstens dreierlei principiell verschiedene Weise die Bewegungen niederster Organismen beeinflussen kann. Nämlich

1) direkt vermittelst Aenderung des Gaswechsels, obne nachweisbare Einmischung einer Empfindung,

2) durch Aenderung der Empfindung des Athembedürfnisses in Folge Aenderung des Gaswechsels,

3) durch Vermittelung eines vermuthlich unserer Lichtempfindung entsprechenden specifischen Processes.

Die drei Processe können combinirt vorkommen, der erste auch allein, oder bloss in Combination mit dem zweiten. Fälle, in denen der erste und dritte, ohne den zweiten, beobachtet werden, habe ich bei grünen Formen bisher noch nicht sicher nachweisen können, halte ihr Vorkommen hier auch für unwahrscheinlich, schon weil die Empfindung des Athembedürfnisses eine viel allgemeinere als die specifische Lichtempfindung ist.

Jeder dieser Processe bängt in anderer Weise von Wellenlänge und Intensität des Lichtes und von den weiteren äusseren Bedingungen ab. Infolge hiervon können die Reaktionen auch beim nämlichen Objekt unter verschiedenen Umständen wesentlich verschieden sein. Heftigste Bewegung und vollkommene Ruhe, Photophobie und Photophilie, gradlinig fortschreitende Bewegung wie Rotation ohne Fortbewegung, Aenderung der Form ohne Ortsveränderung und das Gegentheil werden als Effekt des nämlichen Lichts bei dem nämlichen Individuum unter verschiedenen Bedingungen beobachtet.

Um zu einer genügenden Zergliederung der Erscheinungen zu gelangen, muss die Untersuchung von solchen Fällen ausgehen, in denen man es entweder von vornherein nur mit einem einzigen der genannten Processe zu thun hat oder es wenigstens möglich ist, dieselben gehörig für den Versuch zu isoliren.

Rücksichtlich der drei oben unterschiedenen Wirkungsweisen des Lichtes empfehlen sich für eine solche Untersuchung: Navi- 
cula für den unter 1) Paramaecium bursaria für den unter 2) Euglena viridis für den unter 3) genannten Fall, Organismen, die fast überall in unseren süssen Wässern in Menge vorkommen.

\section{Typus : Navicula.}

Hierzu gehören die meisten beweglichen Diatomaceen wie auch die Oscillarineen. Ihnen schliessen sich im Princip an die chlorophyllhaltenden Zellen mit beweglichem Protoplasma höherer Pflanzen (Vallisneria z. B.). Meine Versuche wurden hauptsächlich mit verschiedenen Arten der Gattungen Navicula und Pinnularia angestellt, die sich alle wesentlich gleich verhielten.

Die Bewegungen dieser Organismen sind an die Anwesenheit freien Sauerstoffs gebunden. Sie hören, mitunter fast augenblicklich, auf, wenn dieser völljg entzogen wird, um zurückzukehren, sobald wieder Sanerstoff geboten wird. Unter Zuhilfenahme der Bakterienreaktion lässt sich dies mit Leichtigkeit nachweisen. Schon bei einer sehr viel niedrigeren Sauerstoffspannung als der normalen wird dabei das Optimum der Bewegung erreicht. Unterhalb dieses steigt und sinkt die Energie ${ }^{1}$ ) der Bewegungen mit der herrschenden Sauerstoffspannung, wie Versuche in der feuchten Kammer mit abwechselnder Einwirkung von Luft, reinem Wasserstoff und Sauerstoff lehrten.

Bekommen sie ans der Umgebung keinen freien Sauerstoff, so können die Naviculaceen sich auf Kosten des Sauerstoffs bewegen, den sie selbst im Licht produciren. Dies muss man daraus folgern, dass das Licht die Bewegungen wieder erweckt, wenn sie im Dunkel in Folge von Sauerstoffmangel aufgehört haben, während bei genügender Sa u erstoffzufuhr von aussen das Licht keinen deutlichen Einfluss auf die Energie der Bewegungen ausübt. Wie bekannt, enthält Navicula gelbes Chromophyll ${ }^{2}$ ), welches im Licht Sauerstoff ausscheidet.

1) Als Maass betrachte ich die mittlere Geschwindigkeit während z. B. einer Minute.

2) Ueber Chromophylle und ihre Beziehungen zur Assimilation vergleiche meinen Vortrag: Lichtabsorptie en Assimilatie in plantencellen. Aanteekeningen van het verhandelde in de sectie-vergad. van het Provinciaal Utrechtsch Genootschap. Utrecht 1882. 
Die Bakterienmethode hat gezeigt, in welcher Weise diese Sauerstoffausscheidung von Wellenlänge und Intensität des Liehts abhängt ${ }^{1}$ ). Dieselbe Abhängigkeit zeigt nun auch die bewegende Wirkung des Lichts.

Mittelst des friuher ${ }^{2}$ ) beschriebenen Mikrospektralobjektivs kann man diese Wirkungen bequem qualitativ und quantitativ untersuchen. Ich theile nur einige der wichtigsten Thatsachen mit.

Wenn eine Navicula im Dunkel resp. Halbdunkel unter dem Deckglas in Folge von 0-Mangel ihre Bewegungen seit einigen Minuten eingestellt hatte ${ }^{3}$ ), so fing sie sofort oder doch innerhalb weniger Sekunden wieder an, sich zu bewegen, sobald sie in den rothen Theil des Mikrospektrums einer Gasflamme gebracht wurde. Verschob ich sie nun ins Grün, Blau oder Violett, so stand sie wieder still und es bedurfte nun einer bedeutenden Erweiterung des Lichtspalts, um sie wieder in Bewegung zn setzen. Die Spaltweite musste für Violett grösser als für Blau, für dieses erheblicher als für Griin sein. Wie Erweiterung des Spalts wirkte Zurïckversetzen ins Roth. Das äusserste sichtbare Roth (vor B) wirkte bedeutend schwächer wiederbelebend als das Roth zwisehen B und C. Ganz unwirksam waren Ultraroth und Ultraviolett.

Dies sind genau dieselben Beziehungen zur Wellenlänge, welche sich mittelst der Bakterienmethode für die $\mathbf{0}$-Ausscheidung der Naviculaceen ergeben haben ${ }^{4}$ ). Auch diese findet nur im sichtbaren Theil des Spektrums statt und zwar betrug im Spektrum des gleichen Gaslichts ihre relative Energie durchschnittlich (in Procenten der maximalen im Roth zwischen B und C)

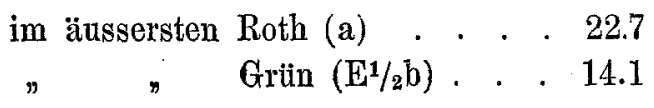

1) Ibid. p. 10 .

2) Dies Archiv, Bd. XXVII, p. 464. 1882.

3) Um den Tropfen hinreichend sauerstofffrei zu bekommen, genügt es, demselben eine an beweglichen Bakterien reiche, übrigens unschädliche, Flüssigkeit zuzusetzen und das Deckglas mit Vaselin oder Fett einzukitten. Die Bakterien absorbiren bald den freien 0 . Da wo ihre Bewegungen gänzlich zur Ruhe gekommen sind, ist der Tropfen genügend 0 -frei und hören auch im Dunkel oder Halbdunkel die Bewegungen der Naviculareen bald oder sogleich auf.

4) Lichtabsorptie en assimilatie u. s. w., 1.c. p. 10. Siehe auch Botan. Zeitg. Nr. 26, 1882 und einen demnächst daselbst folgenden Aufsatz. 


$$
\begin{array}{ccccccc}
\text { im äussersten } & \text { Blau }(\mathrm{F}) & . & . & . & . & 6.9 \\
& \| & \text { Violett }(\mathrm{G}) & . & . & . & 1.2
\end{array}
$$

Bewegte sich eine Navicula zufällig über die Grenze des Lichts ins Dunkel, oder aus dem rothen in den grünen Theil des Mikrospektrums, so blieb sie da bald unbeweglich liegen, wenn sie nicht etwa ihre Bewegungsrichtung änderte und dabei ins Licht, bezüglich in das Roth zurïckkam. Letzteres geschah jedoch in einer verhältnissmässig so geringen Anzahl von Fällen, dass es ausschliesslich als Zufall betrachtet werden muss und nicht als Beweis für ein die Bewegungen regulirendes Unterscheidungsvermögen der Zelle aufgefasst werden darf. Auch diese Versuche wurden natürlich in möglichst sauerstofffreien Tropfen angestellt.

Die Intensität des zum Erwecken der Bewegungen erforderlichen Lichtes war in allen Fällen von gleicher Ordnung mit der, welche zur Erzeugung deutlicher, mittelst empfindlicher Bakterien nachweisbarer O-Ausscheidung erforderlich war. In der Regel schien sie etwas geringer zu sein, doch kam auch das Gegentheil vor und jedenfalls waren die Differenzen unbedeutend im Vergleich zu den durch die verschiedene Empfindlichkeit der Individuen bedingten. Der Schwellenwerth der Lichtstärke war auch für Roth zwischen $\mathrm{B}$ and $\mathrm{C}$ durchschnittlich viel bedeutender als der entsprechende Werth für das Auge. Mässige Beleuchtung wirkt also im Allgemeinen noch wie völlige Dunkelheit, was für die Beobachtung, namentlich mit starken Vergrösserungen, selbstverständlich grosse praktische Vortheile mit sich bringt.

\section{Typus: Paramaecinm bursaria.}

Verwickelter und von höherer Ordnung sind die Lichtwirkungen bei dem grünen Pantoffelthierchen und anderen chlorophyllhaltigen Ciliaten (Stentor viridis, Bursaria u. a.). Hier ist ohne die Annahme eines die Bewegungen regulirenden Empfindungsvermögens nicht auszukommen, wennschon von Lichtperception im gewöhnlichen engeren Sinne, von Sehen, noch keine Rede sein kann.

Der richtige Weg zu näherer Einsicht wird auch hier gewiesen durch Priffung der Wirkung des Lichts bei verschiedenen Sauerstoffspannungen. 
Bei normalem oder etwas höherem Sauerstoffgehalt des Wassers sind die Thierchen meist sehr ruhig. Lange Zeit, oft minutenlang, bleiben sie auf derselben Stelle stehen. Sie reagiren dann durchaus nicht auf Licht ${ }^{1}$ ).

Sinkt die Sauerstoffspannung nur wenig unter die Norm, so werden sie unruhig und suchen an Stellen zu gelangen, wo sie mehr 0 finden. Hat man die Paramäcien z. B. eine Zeit lang ${ }^{2}$ ) im Wassertropfen unter einem mässig grossen, nicht verkitteten Deckglas im Dunkeln gehalten, so findet man sie nachher dicht am Rand des Deckglases, oder, falls grössere Luftblasen im Tropfen vorhanden, um diese angesammelt. Hier pflegen sie dann ruhig, wie im unbedeckten Tropfen, zu stehen. Wird der Tropfen unter ubbrigens ganz gleichen Bedingungen im Licht aufbewahrt (diffuses, nicht zu schwaches Tageslicht geniigt), so kommt es nicht zu jenen Ansammlungen, sondern die Thierchen halten sich, wie in 0-reichen Tropfen, äberall unter dem Deckglas mit anscheinend gleichem Behagen auf. Zuweilen, namentlich bei schwächerer Belenchtung stehen sie hier und da in kleinen Gruppen beisammen.

Nimmt der Sanerstoffdruck bedeutend ab, indem man z. B. reinen Wasserstoff in genügend starkem Strome über den unbedeckt in der feuchten Gaskammer hängenden Tropfen hinführt, oder bei längerem Verweilen des Präparats im Dunkeln unter eingekittetem Deckglas, so schwimmen die Paramäcien bald sehr unruhig bin und her, endlich mit ziemlich grosser und gleichmässiger Geschwindigkeit in gerader Richtung vorwärts, unter beständiger Rotation um die Längsachse ihres allmählich aus der normalen platten in eine mehr gestreckt ellipsoidische Form übergehenden Körpers. Wenn man sie jetzt einige Minuten lang fortwährend stark beleuchtet, am besten mit weissem oder rothem Licht, so

1) Selbstverständlich gilt dies nur für nicht ïbermässige Beleuchtungsintensitäten. Concentrirtes Sonnenlicht kann durch Erhitzung leicht störend einwirken.

2) Die Dauer dieser Zeit hängt selbstverständlich von den Dimensionen des Tropfens und der Masse und Art der denselben bevölkernden 0-consrmirenden Organismen ab. Man kann sie auf wenige Minuten reduciren, wenn man dem Tropfen etwas Flüssigkeit mit gut beweglichen Fäulnissbakterien zusetzt. 
werden sie wieder ruhig und platt. In diesem Zustand nun, bei anhaltend ungenügender Sauerstoffzufuhr von aussen, reagiren sie sehr lebhaft und scharf auf Aenderungen der Beleuchtung.

Ueberschreiten sie z. B. zufällig die Grenze von Licht und Dunkel ${ }^{1}$ ), oder tauchen sie auch nur mit der vorderen Hälfte ihres Leibes eine Strecke weit in das Dunkel ein, so kehren sie sofort um nach dem Licht, wie wenn das Dunkel ihnen unangenehm wäre.

Im Mikrospektrum von Gaslicht gehen sie aus Grün und Blau nach dem Roth, am liebsten nach Roth von etwa $0.65-0,70 \mu$ Wellenlänge ( $\mathrm{B}-\mathrm{C}$ Fraunhofer). Hier bleiben sie, bei genügender Lichtstärke, still stehen, wie unter gewöhnlichen Umständen im 0-haltigen Tropfen. Verschiebt man jetzt Objektträger oder Spektrum, so dass sie in Licht grösserer oder geringerer Wellenlänge kommen, so werden sie alsbald unruhig. Je bedeutender die Aenderung der Wellenlänge, um so schneller erfolgt die Reaktion: im äussersten Fall scheinbar momentan, unter anderen Umständen aber auch erst nach 5-10 Sekunden und darïber. Auch bei ganz allmählicher langsamer Veränderung tritt schliesslich Unruhe ein. Hierbei scheint aber einige Akkomodation stattfinden zu können. Verstärkung des Lichts oder Rückkehr ins erste Roth beruhigt die Thiere wieder u. s. f. Ultraroth und Ultraviolett wirken stets wie Dunkelheit. Violett wirkt schwächer wie Blau (F), dieses kaum schwächer, im Mikrospektrum von Sonnenlicht selbst etwas stärker wie Grün (E-b), auch Gelb und Orange, sowie das äusserste sichtbare Roth noch viel schwächer wie Roth zwischen $\mathrm{B}$ und $\mathrm{C}$.

Offenbar liegt die Erklärung dieser Erscheinungen darin, dass die ungenügende Sauerstoffufuhr von aussen durch die Sauerstoffentwicklung der innen, im Mesoplasma gelegenen Chlorophyllkörper compensirt werden kann. Schon vor

1) Das Präparat befand sich bei allen diesen Versuchen in meinem dunklen Mikroskopirkasten (Dies Archiv, Bd. XXIII, p. 577). Ein scharfbegrenzter Lichtbezirk ward im übrigens ganz dunkeln Tropfen mittelst des Mikrospektralapparates oder eines früher (Dies Archiv, Bd. XXVI, p. 538 Anmerk.) erwähnten Diaphragmenapparates und der Abbe'schen Condensorlinse erzengt. 
mehr als einem Jahre habe ich mittelst der Bakterienmethode nachgewiesen ${ }^{1}$ ), dass diese Körper und ebenso die entsprechenden Gebilde anderer niederer Thiere (Hydra, Spongilla) wirklich wie ächte Chlorophyllkörner funktioniren, d. h. im Licht Sauerstoff abscheiden ${ }^{2}$ ). Die Sauerstoffabscheidung des Chlorophyll zeigte dieselbe Abhängigkeit von Wellenlänge und Stärke des Lichts wie die beschriebene Wirkung des Lichts auf die Bewegungen der Paramäcien ${ }^{3}$ ). Ihre Energie betrug durchschnittlich (in Procenten des maximalen, bei B-C gelegenen, Werthes) im Mikrospektrum von Sonnenlicht Gaslicht

$\begin{array}{cclrr}\text { im äussersten Roth (a) } & 9,7 & 24.7 \\ " & " & \text { Gelb (D) } & 35.2 & 23.3 \\ " & " & \text { Grün (E匚/2b) } & 14.6 & 6.2 \\ " & " & \text { Blau (F) } & 25.5 & 5.3 \\ " & " & \text { Violett (G) } & 8.2 & 0.8\end{array}$

Ehenso wie Sauerstoffmangel beunruhigt andererseits auch bedeutende Erhöhung der Sauerstoffspannung die Paramäcien in sebr auffälliger Weise. Namentlich haben sie dann die Neigung, in gerader Richtung oder in starkem Bogen weitaus rückwärts zu schwimmen, überhaupt von den Orten höherer Sauerstoffspannung zuriickzuweichen. Derselbe Erfolg nun zeigt sich, wenn man bei schon ziemlich, aber nicht hinreichend über die normale erhöhter Sauerstoffspannung, z. B. während des Durchleitens eines Stroms reinen Sauerstoffs bei gewöhnlichem Druck durch die feuchte Kammer, die Thierchen plötzlich stark beleuchtet. Anstatt ruhig zu werden, wie im gleichen Fall bei O-Mangel, werden die erst noch ziemlich normalen Bewegungen dann augenblicklich höchst ungestüm. Oft schiessen die Paramäcien auf einmal pfeilschnell weit rückwärts um erst im Dunkeln allmählich wieder ruhiger zu werden. Neues starkes Beleuchten weckt neue Unruhe u. s. f. Anstatt des Dunkels fliehen sie jetzt das Licht. Die Grenze beider passiren sie nur in der Richtung von Hell zu Dunkel,

1) Proc. verb. k. Akad. v. wetensch. te Amsterdam. Afd. Natuurk. Zitting v. 28. Mei 1881. S. auch dies. Archiv, Bd. XXV, 1881, p. 285.

2) Ob sie morphologisch Chlorophyllkörnern gleichwerthig, oder Symbionten sind, wie Semper vermuthete und Géza Entz und Brandt wahrscheinlich gemacht haben, thut natürlich hier nichts zur Sache.

3) Vgl. Lichtabsorptie en assimilatie u. s. w., l. c. p. 10. 
nicht in der umgekehrten. Denn wie bei O-Mangel an der Grenze des Dunkels, so scheuen sie jetzt an der Grenze des Lichts zurïek. In jenem Falle photophil, sind sie in diesem photophob.

In Uebereinstimmung mit der gegebenen Erklärung fliehen sie jetzt nun anch am meisten das rothe Licht, die andern Wellenlängen in dem Maasse weniger, als die assimilatorische Wirkung derselben eine geringere ist.

Wie aus Allem hervorgeht, haben die Paramäcien ein sehr feines Empfindungsvermögen für Unterschiede der Sanerstoffspannung and ist es dies Empfindungsvermögen, durch dessen Vermittlung das Licht die Bewegungen beeinflusst.

\section{Typus: Euglena viridis.}

Wiederum ganz anderer Art ist der physiologische Mechanismus, mittelst dessen Euglena viridis, auch Colacium, Trachelomonas und andere verwandte Formen, auf Licht reagiren. Dies geht schon daraus hervor, dass hier die Reaktion in hohem Grade unabhängig ist von der Sauerstoffspannung. Sowohl bei Sauerstoffmangel wie bei normaler oder ultranormaler Sauerstoffspannung sind nämlich die Euglenen für Licht sehr empfindlich.

Im Dunkeln, bei sehr hochgradigem Sauerstoffmangel, gehen sie natürlich allmählich zu Grunde und werden also auch die Reaktionen auf Licht schwächer und unregelmässiger. Aber sie erfolgen doch noch zu einer Zeit, wo die normalen Bewegungen schon sehr unruhig, taumelnd geworden sind. Auch bei sehr hoher Sauerstoffspannung scheint die Empfindlichkeit für Licht stets etwas geringer zu sein als in der Norm. Namentlich unmittelbar nach plötzlicher Erhöhung der O-Spannung ist dies meist deutlich der Fall. Zwischen diesen Extremen hat aber der Sauerstoffdruck weder in qualitativer noch in quantitativer Hinsicht nennenswerthen Einfluss. Die maximale Empfindlichkeit seheint indessen in der Regel bei etwas höherem als normalem 0-Druck zu bestehen.

Die Erscheinungen sind gewöhnlich die folgenden. Bei partieller Erleuchtung des Tropfens ${ }^{1}$ ) häufen sich die Euglenen allmählich in dem Lichtbezirke an. Dieser wirkt wie eine Falle,

1) Ueber die Methodik s. p. 393, Anmerk. 1. 
denn einmal hineingekommen, gehen die Euglenen in der Regel nicht wieder heraus. Sie kehren an der Grenze des Dunkels immer sogleich wieder um ins Helle. Falls sie, was bei schnellem Vorwärtsschwimmen wohl einmal geschieht, ganz ins Dunkel hineingekommen sind, sistiren sie doch sofort die weitere Vorwärtsbewegung, drehen um eine ihrer kurzen Axen, probiren - oft unter bedeutenden Gestaltsänderungen - in verschiedenen Richtungen fortzukommen, bis sie endlich wieder ins Licht gerathen. Seltner verirren sie sich weiter ins Dunkel.

Folgt schon aus der grossen Unabhängigkeit von der herrschenden Sauerstoffspannung, dass die beschriebenen Reaktionen nicht auf Aenderungen der, übrigens sehr energischen, 0 -produktion im Chlorophyll der Englenen beruhen, auch längs eines anderen mehr direkten Weges lässt sich dies zeigen.

Wie bekannt, ist der vorderste Abschnitt des beim Schwimmen langgestreckt spindelförmigen Körpers der Euglenen meist chlorophyllfrei. Hier entspringt aus dem farblosen Protoplasma die lange Geissel und liegt der rothe Pigmentfleck. Wenn man nun einen scharfen Schatten (durch passende Bewegung des Spiegels oder des Spektralobjektivs, oder auch des Objektträgers) von hinten her uiber eine im Licht gerade vorwärts schwimmende Euglena vorrücken lässt, dann reagirt das Thier nicht, solange nicht der vorderste chlorophyllfreie Abschnitt mit dem Pigmentfleck ins Dunkel getaucht wird. Im Augenblick aber, wo dies geschieht, stockt die Bewegung, die Euglena kehrt um, verkürzt sich auch wohl, kurz benimmt sich gerade so wie wenn ihr ganzer Körper plötzlich verdunkelt worden wäre. Ob die Geissel mit in den Schatten kommt ist gleichgiltig. Eine direkte Wirkung des Lichtes anf die Wimper, die Hofmeister für andere Fälle vermuthete, besteht hier also nicht.

Achtet man genau auf das was geschieht, wenn eine Euglena, das orale Ende voraus, die Grenze zwischen Licht und Dunkel in der Richtung nach dem letzteren zu eben ïberschreiten will, so bemerkt man, dass die Reaktion meist erst nach dem Moment beginnt, in welchem die Gegend des im farblosen Vorderende gelegenen Pigmentflecks ins Dunkel eintaucht. Die Reaktion (Umkehr, Verkürzung u. s. w.) erfolgt dann oft so schnell, dass das Chlorophyll des Körpers nicht einmal den Rand des Dunkels berührt. Die Lichtpereeption von Euglena 
hat also ausschliesslich am chlorophyllfreien Vorderende des Körpers ihren Sitz. Meine anfängliche Vermuthung aber, dass der Pigmentfleck die Stelle näher anweise, an der die Lichtperception zu Stande lrommt, hat sich nicht bestätigt. Bei sehr grossen, langsam schwimmenden Euglenen konnte ich neverdings wiederholt ganz sicher constatiren, dass die Reaction erfolgte, noch ehe der Pigmentfleck ins Dunkel tauchte. Es ist also das farblose durchsichtige Protoplasma am vorderen Körperende der Ort, an welchem die primäre Erregung durch Licht stattfindet. Trotzdem wäre es, wie mir scheint, voreilig, dem Pigmentfleck jede Beziehung zur Lichtempfindung abzusprechen. Er könnte ja in ähnlicher Weise wie bei höheren Thieren die Pigmentschicht der Retina functioniren, etwa durch Mitwirkung bei der Bereitung lichtempfindlicher Substanzen. Immerhin mag man einstweilen fortfahren, ihn Augenfleck zu heissen.

Ob die hier ermittelten Beziehungen bei allen mit einem sogenannten Augenfleck versehenen Formen bestehen, wird sich schwer entscheiden lassen. Kleinheit des Körpers, zu grosse Geschwindigkeit der Bewegungen, ungünstige Lagerung des Pigmentflecks bieten oft unüberwindliche Schwierigkeiten. Uebrigens wird man eine besondere Ausbildung des Empfindungsvermögens am oralen Körperpol wohl bei allen diesen niederen Organismen vermuthen dürfen. Findet sich dieselbe doch, aus naheliegenden Gründen, allgemein bei Thieren. Auch bei Paramaecium bursaria ist beiläufig die vordere Hälfte des Körpers, von der Umgebung der hier ziemlich weit hinten, auf der Bauchseite gelegenen Mundöffnung an, empfindlicher gegen die durch Wechsel der Beleuchtung hervorgerufenen Schwankungen der $\mathrm{O}$-spannung als die hintere Körperhälfte. Letztere muss trotz grösseren Chlorophyllgehalts längere Zeit beschattet werden, ehe eine Reaction erfolgt, erstere - auch schon das vorderste Achtel des Körpers - unter gleichen Verhältnissen nur einen Augenblick. Eine direkte Einwirkung des Lichts ohne Vermittlung der Chlorophyllkörper, auf das farblose Plasma scheint hier aber nicht za bestehen. Ich erhielt wenigstens nie eine unzweifelhafte Reaktion, wenn ich, was bei vielen Individuen möglich, nur den vordersten chlorophyllfreien Körperrand mit den darin enthaltenen Trichocysten, in einer Breite von etwa $0,01 \mathrm{~mm}$ erleuchtete bezüglich beschattete. Sobald aber einige 
Chlorophyllkörper an dieser Stelle mitgetroffen wurden, erfolgte die charakteristische Bewegung.

Wie darchaus versehieden die Vorgänge bei Paramaecium und Euglena sind, zeigt sich auch weiter, wenn man das Verhalten beider gegen Strahlen verschiedener Wellenlänge vergleicht.

Im Mikrospektrum von Tag- und Gaslicht häufen sich die Euglenen zwar auch nur im sichtbaren Theil an, aber anstatt das Roth zu suchen, haben sie - und zwar bei sehr grosser wie bei geringer Lichtstärke und schon bei sehr mässiger Reinheit des Spektrums (grosse Spaltweite) - die Neigung sich an der brechbaren Seite anzusammeln. Bei allmählicher Verengerung des Spalts concentriren sie sich mehr und mehr im Blau, endlich, bei einer bestimmten, ziemlich geringen Lichtstärke und erheblicher Reinheit des Spektrums nahezu ausschliesslich auf einem schmalen Streifen ungefähr an der Stelle der Fraunhofer'schen Linie F, zwischen den Wellenlängen 0,47 and $0,49 \mu$. Hier fahren sie fort, sich in normaler Weise zu bewegen, kehren aber immer an der Grenze von Dunkel, von Grün, die meisten auch an der Grenze von Indigo oder Violett wieder um.

Wird das Bild des Spektrums mittelst einer ins Ocular gelegten Glastheilung in Felder von gleicher Breite getheilt, so kann man die unter den genannten Umständen in den einzelnen Feldern vorhandenen sehr verschiedene Mengen von Individuen leicht zählen. So fand ich in einem von vielen in allem Wesentlichen übereinstimmenden Fällen in

$$
\begin{aligned}
& \text { Feld } 1 \text { (A bis } \mathrm{C}^{3} / 4 \mathrm{D} \text { ) : } 2 \text { Individuen, } \\
& \text { "2 }\left(\mathrm{C}^{3} / 4 \mathrm{D}-\mathrm{D}^{5} / 6 \mathrm{E}\right): 0 \text { " } \\
& \text { "3( } 3\left(\mathrm{D}^{5} / 6 \mathrm{E}-6^{5 / 6} \mathrm{~F}\right): 16 \text {, } \\
& \text { "4 }\left(6^{5} / 6 \mathrm{~F}-\mathrm{F}^{4} / 7 \mathrm{G}\right): 100 \quad \text { " } \\
& " 5\left(\mathrm{~F}^{4} / 7 \mathrm{G}-\mathrm{G}\right) \quad: 24 \text { " } \\
& \text { "6(G-G } \left.\mathrm{G}^{1} / 2 \mathrm{H}\right) \quad: \quad 3 \text { " }
\end{aligned}
$$

Die 16 Individuen in Feld 3 befanden sich alle an der Seite von Feld 4 (zwischen Wellenlänge 0,45 und $0,50 \mu$ ), ebenso die meisten in Feld 5 (zwischen Wellenlänge 0,47 und $0,46 \mu$ ). Die Lichtquelle war in diesem Falle eine starke Glasflamme ${ }^{1}$ ) (Sugg's

1) Vergl. über dieselbe und ihr Verhältniss zum Sonnenlicht die quantitativen Angaben in dem oben citirten Vortrag: "Lichtabsorptio u. s. w." 1. c. p. 11. 
Brenner). Das Mikrospektrum wurde mittelst Objektiv A von Zeiss entworfen und durch Objektiv B mit Ocular 3 (eingeschobener Tubus) betrachtet. Die Spaltweite betrug 0,42 mm. Der Spalt konnte noch sehr viel weiter verschmälert werden, ohne dass das Bild sich wesentlich verändert hätte. Endlich aber schwammen die Euglenen in allen Richtungen auseinander, wie nach völliger Verdunkelung.

Wie leicht einzusehen, kann die Empfindlichkeit der Euglenen für Unterschiede der Wellenlänge und für Intensitätsunterschiede an jedem Punkte des Spektrums mit Hilfe des Mikrospektralobjektivs gemessen werden. Ich will in dieser Beziehung nur noch bemerken, dass der kleinste für Euglena merkbare Intensitätsuntersehied für Roth, Gelb und Grün ansehnlich grösser ist als für Blau (zwischen 0,47 und $0,49 \mathrm{~m}$ Wellenlänge) und auch für Violett. Die Schwellenwerthe der Intensität sind im Allgemeinen ziemlich hoch, sehr bedeutend höher jedenfalls wie für das menschliche Auge.

Farbenblinde Euglenen babe ich bisher ebensowenig finden können wie völlig blinde. Doch zeigen Euglenen von verschiodenen Lokalitäten und Entwickelungsstufen oft beträchtliche quantitative Unterschiede der Empfindlichkeit. Interessant würde es sein, das Verhalten santoninisirter Euglenen im Mikrospektrum zu untersuchen, wie denn üherhaupt eine Menge für die vergleichende Physiologie des Sehens wichtiger Frágen auf der Hand liegen, deren Beantwortung keine wesentlichen Schwierigkeiten bieten wird.

Ich bin keineswegs der Meinung, dass die beschriebenen drei Typen lichtempfindlicher Organismen bereits alle hier in $\mathrm{Be}-$ tracht kommenden Wirkungsweisen des Lichts repräsentiren. Ohne Zweifel kann das Licht - ganz abgesehen von thermischen Wirkungen - auch noch durch Vermittlung anderer als der hier behandelten Processe die Bewegungen vieler einfacher Organismen beeinflussen. Hierauf weisen u. a. manche der von Stras burger in seiner bekannten, reichhaltigen Schrift ${ }^{1}$ ) gesammelten Thatsachen. Ebenso auch die merkwürdigen Reaktionen des unlängst

1) E. Strasburger, Wirkung des Lichtes und der Wärme auf Schwärmsporen. Jena 1878. 
von mir entdeckten Bacterium photometricum ${ }^{1}$ ), iber welche ich in einem folgenden Aufsatz ausführlicher berichten werde. Die vorliegenden Mittheilungen sollen nur der Forschung einige leitende Fäden bieten, deren Verfolgung für die fernere Orientirung auf dem bisher so dunklen, umfangreichen Gebiete von Nutzen zu sein verspricht.

\section{Ueber den von 0 . Loew und Th. Bokorny erbrachten Nachweis von der chemischen Ursache des Lebens.}

Von

\section{E. Baumann.}

In dem kuirzlich erschienenen Heft 1 und 2 des 28. Bandes dieses Archivs führen 0 . Loew und Th. Bokorny Klage über meine Besprechung ihrer Schrift: „Ueber die chemischen Ursachen des Lebens, theoretisch und experimentell nachgewiesen, München $1881^{\text {", }}$, in der Deutschen Literaturzeitung vom 22. April 1882. L. und B. erklären die in dieser Besprechung enthaltene Kritik, welche die Spekulationen und die Schlüsse ihrer Schrift seharf verurtheilt, für unbegrïndet und fordern mich anf, sachliche Einwände gegen die von ihnen vertretenen Behauptungen vorzubringen. Dieser Aufforderung entspreche ich um so bereitwilliger, als es einerseits nicht möglich ist, in einer Besprechung, deren Umfang auf einen kleinen Raum beschränkt ist, auf die Ansichten der Verfasser im einzelnen, und namentlich die Art ihrer Beweisführung und Schliusse zu ziehen einzugehen, andererseits weil ich glaube, dass Publikationen wie die vorliegende von $L$. und B., die schon vermöge ihres Titels das allgemeinste Interesse erregen müssen, nicht mit Stillschweigen übergangen werden dürfen, sondern, wie es auch L. und B. wünschen, eine eingehende kritische Besprechung verdienen.

1) Over een nieuw voor licht gevoelig bacterium. Proc. verb. d. k. Akad. van wetenschappen. Amsterdam. Afd. Natuurk. Zitting van 25. Maart 1882. 